\title{
IMPACTO SOCIAL DO USO ABUSIVO DE DROGAS PARA DEPENDENTES QUÍMICOS REGISTRADOS EM PRONTUÁRIOS
}

\author{
Fernanda Carolina Capistrano ${ }^{1}$, Aline Cristina Zerwes Ferreira², Mariluci Alves Maftum³, \\ Luciana Puchalski Kalinke ${ }^{4}$, Maria de Fátima Mantovani ${ }^{5}$
}

\begin{abstract}
RESUMO: Pesquisa descritiva transversal realizada em uma unidade de reabilitação para dependentes químicos. O objetivo foi identificar o impacto social do uso abusivo de drogas para dependentes químicos e os dados foram coletados em 350 prontuários referentes ao ano de 2010. Os resultados demonstraram que $60 \%$ dos dependentes possuíam idade entre 20 e 39 anos, o diagnóstico clínico de maior prevalência foi transtornos mentais e comportamentais devido ao uso de múltiplas drogas e de outras substâncias psicoativas com início precoce. Decorrentes da dependência química, 54\% dos casos tiveram problemas relacionados à família, $29,4 \%$ conflitos conjugais, $63,2 \%$ prejuízos laborais, $20,6 \%$ cometeram infração penal e $26,6 \%$ praticaram violência. Conclui-se que a dependência química transforma os relacionamentos familiares, causa prejuízos laborais e sociais, contribui para o desenvolvimento da delinquência e da violência sendo necessário adequar e investir em políticas públicas para a reestruturação desses sistemas. DESCRITORES: Enfermagem; Saúde mental; Transtornos relacionados ao uso de substâncias.

\section{THE SOCIAL IMPACT OF THE ABUSIVE USE OF DRUGS FOR ADDICTS REGISTERED IN MEDICAL RECORDS}

\begin{abstract}
This descriptive, cross-sectional research was undertaken in a rehabilitation center for drug addicts. The objective was to identify the social impact of the abusive use of drugs for drug addicts. The data was collected in 350 medical records referent to the year 2010. The results showed that $60 \%$ of the addicts were aged between 20 and 39 years old, and that the most prevalent clinical diagnosis was mental and behavioral disorders due to the use of multiple drugs and other psychoactive substances with early onset. As a result of the addiction to drugs, $54 \%$ of the cases had problems related to their families, $29.4 \%$ had marital conflicts, $63.2 \%$ had problems with work, $20.6 \%$ had broken the law and $26.6 \%$ practised violence. It is concluded that drug addiction transforms family relationships, causes social and work problems, and contributes to the development of delinquency and violence, it being necessary to adapt, and invest in, public policies for restructuring these systems. DESCRIPTORS: Nursing; Mental health; Disorders related to substance use.

\section{IMPACTO SOCIAL DEL USO ABUSIVO DE DROGAS PARA DEPENDIENTES QUÍMICOS REGISTRADOS EN PRONTUARIOS}

RESUMEN: Investigación descriptiva transversal realizada en una unidad de rehabilitación para dependientes químicos. El objetivo fue identificar el impacto social del uso abusivo de drogas para dependientes químicos y los datos fueron obtenidos en 350 prontuarios referentes al año de 2010. Los resultados apuntan que el 60\% de los dependientes tenían edad entre 20 y 39 años; el diagnóstico clínico de mayor prevalencia fue el de trastornos mentales y de comportamiento a causa del uso de múltiplas drogas y de otras sustancias psicoactivas con inicio precoz. A causa de la dependencia química, $54 \%$ de los casos presentaron problemas relacionados a la familia, 29,4\% conflictos conyugales, 63,2\% daños laborales, $20,6 \%$ incurrieron en faltas penales y $26,6 \%$ practicaron violencia. Se concluiye que la dependencia química cambia las relaciones familiares, causa daños laborales y sociales, contribuye para el desarrollo de la delincuencia y de la violencia siendo necesario adecuar y promover políticas públicas para la restructuración de esos sistemas.

DESCRIPTORES: Enfermería; Salud mental; Trastornos relacionados al uso de sustancias.

\footnotetext{
${ }^{1}$ Acadêmica de Enfermagem da Universidade Federal do Paraná - UFPR. Membro do Núcleo de Estudos, Pesquisa e Extensão em Cuidado Humano em Enfermagem - NEPECHE. Bolsista voluntária de Iniciação Científica.

${ }^{2}$ Acadêmica de Enfermagem da UFPR. Membro do NEPECHE. Bolsista CNPq de Iniciação Científica.

${ }^{3}$ Enfermeira. Doutora em Enfermagem. Professora do Departamento de Enfermagem e do Programa de Pós-Graduação em Enfermagem - PPGENF UFPR. Coordenadora do PPGENF UFPR e Vice-Coordenadora do NEPECHE.

${ }^{4}$ Enfermeira. Doutora em Ciências da Saúde. Professora do Departamento de Enfermagem e do PPGENF UFPR. Vice-coordenadora do PPGENF UFPR. Membro do Grupo de Estudos Multiprofissional em Saúde do Adulto - GEMSA.

${ }^{5}$ Enfermeira. Doutora em Enfermagem. Professora do Departamento de Enfermagem e do PPGENF UFPR. Membro do GEMSA.
} 


\section{INTRODUÇÃO}

A dependência química já foi considerada pela sociedade um desvio de personalidade que acometia aqueles com dificuldades de relacionamento. No entanto, ao analisar as dimensões biológicas, sociais e culturais do ser humano, este conceito torna-se inapropriado. Atualmente, compreende-se que não se trata somente de uma questão de escolha, a qual depende da razão ou da moralidade de cada indivíduo ${ }^{(1-2)}$.

A dependência química ocasiona uma síndrome composta por um conjunto de fenômenos fisiológicos, comportamentais e cognitivos que alteram os valores pessoais, familiares e sociais. Caracteriza-se em um estado de uso, compulsivo e incontrolável, da substância psicoativa, quase sempre, associado a sofrimento clínico, ocupacional ou social que gera prejuízos em diversas esferas da sua vida ${ }^{(2)}$.

Estimativas mundiais apontam que aproximadamente 230 milhões de pessoas, com idade entre $15 \mathrm{e}$ 64 anos, usam drogas ilícitas a cada ano. Destes, 27 milhões ou $0,6 \%$ da população mundial apresentam problemas decorrentes desta prática. Entrementes, as substâncias psicoativas ilícitas matam 0,2 milhões de pessoas a cada ano, favorecendo o devastamento familiar e a miséria de milhares de pessoas ${ }^{(3)}$.

Entre os diversos motivos que levam o indivíduo a usar drogas, destaca-se a grande dificuldade de suportar as responsabilidades sociais que lhe cabem. Geralmente, estes renunciam a necessidade e a esperança de modificar o meio social que convive ${ }^{(4)}$.

Ao se tornar um dependente químico, seu comportamento passa a ser condicionado, na maior parte do tempo, na busca e na aquisição da droga, perdendo, muitas vezes, o interesse no que antes julgava importante. A redução dos cuidados pessoais e a perda de envolvimento social acentua esta condição ${ }^{(1-2)}$.

Quando a dependência se instala, os indivíduos não relacionam os problemas de aspectos biológicos, social e ocupacional com o uso de drogas, ou não conseguem perceber esta relação e minimizam as consequências ou negam o estado cronificado ${ }^{(2,4)}$. Destarte, não é adequado considerar somente os critérios a nível cerebral da dependência de drogas, isso porque o contexto social que este transtorno se desenvolve e se manifesta são de extrema importância, pois são os estímulos condicionantes da dependência ${ }^{(5)}$.

Um núcleo familiar instável e fragilizado no qual não há identificação saudável do indivíduo com os pais, a dificuldade de manejo com conflitos cotidianos demonstrando sentimentos de impotência devido à impossibilidade em manter um projeto de vida e a baixa tolerância à frustrações são exemplos de tais estímulos, comumente associados ao processo de dependência ${ }^{(4)}$.

A dependência química ocasiona prejuízos em diversos sistemas, entre eles o familiar, com o adoecimento das relações, principalmente com cônjuges e filhos; nos estudos com a interrupção; o laboral com absenteísmo, atrasos e incapacidade de realizar as tarefas que refletem na sociedade como um todo ${ }^{(4)}$. Esta situação acarreta altos custos para a sociedade, pois, mesmo diante dos problemas ocasionados pela dependência, os mesmos continuam a consumir drogas ${ }^{(1,5)}$. Destarte, a recuperação do dependente está diretamente relacionada à reestruturação desses sistemas em suas vidas $^{(5-6)}$.

Há que se considerar que o uso de drogas é muito mais do que uma decisão do indivíduo, que muitas vezes se expõe em diversas situações, é também uma confluência de fatores que se interrelacionam, caracterizando a síndrome do comportamento de risco. Então é precoce e inapropriada a ideia de que somente um fator isolado é necessário para induzir o consumo ${ }^{(6)}$.

Compreende-se que a dependência de substâncias psicoativas é um transtorno mental e comportamental no qual a heterogeneidade prepondera, pois afeta variadas pessoas, de diferentes modos e por várias razões e circunstâncias. Portanto, conhecer o impacto social ocasionado pelo uso abusivo de drogas favorece o manejo ao dependente e contribui para o desenvolvimento de um cuidado específico e condizente à real necessidade dessa clientela incluindo ações de prevenção ao consumo de drogas.

Este artigo teve como objetivo caracterizar o impacto social do uso abusivo de drogas para dependentes químicos.

\section{MÉTODO}

Pesquisa descritiva transversal desenvolvida em uma unidade de reabilitação para dependentes químicos na região metropolitana de Curitiba.

A coleta de dados ocorreu de dezembro de 2010 a fevereiro de 2011 através de 350 prontuários de todos os pacientes que estiveram em tratamento no período da coleta de dados. Utilizou-se formulário estruturado com variáveis preditoras que visavam à caracterização dos pacientes (idade, início de uso, diagnóstico clínico) e as variáveis de desfecho (prejuízos familiares, ocupacionais, sociais e o envolvimento com a criminalidade e violência). 
Os dados coletados foram identificados por códigos numéricos e organizados em uma planilha Excel ${ }^{\circledR}$ 2007. Posteriormente, na análise das informações empregou-se a análise estatística descritiva por intermédio do programa computacional Static $®$. Os resultados foram obtidos por meio de cálculos relativos, a partir do total de dados válidos, haja vista que alguns prontuários apresentavam informações incompletas.

Esta pesquisa integra o projeto $O$ reinternamento de dependentes químicos em uma unidade de reabilitação: causas e motivações da recaída aprovado no Comitê de Ética do Setor de Ciências da Saúde da Universidade Federal do Paraná - UFPR, em reunião do dia 28 de abril de 2010, sob a inscrição CEP/SD: 904.029.10.03; CAAE: 0825.0.000.091-10 e respeita os aspectos éticos de acordo com a Resolução n.196/96, regente à época doo estudo.

\section{RESULTADOS}

Os resultados obtidos da amostra composta de 350 prontuários de dependentes químicos em tratamento na unidade de reabilitação estudada foram caracterizados da seguinte forma: pessoas com idade entre 20 e 39 anos compunham $60 \%(n=210)$ da amostra, o diagnóstico clínico de maior prevalência foi o de transtornos mentais e comportamentais devidos ao uso de múltiplas drogas e ao uso de outras substâncias psicoativas (F-19) em 49,4\% $(n=173)$, seguido por transtornos mentais e comportamentais devido ao uso de álcool (F-15) 42,9\% $(\mathrm{n}=150)$ com início precoce, em média de 17,1 anos $(\mathrm{n}=350)$.

Quanto às perdas decorrentes da dependência química, (Tabela 1), 6,6\% $(\mathrm{n}=23)$ dos usuários abandonaram os estudos ao menos uma vez na vida e $54 \%$ (n= 189) tiveram problemas relacionados à família. Quanto aos problemas conjugais, $29,4 \%(n=103)$ tiveram algum conflito, sendo que desses, 91,6\% $(\mathrm{n}=87)$ evoluíram para a separação conjugal em decorrência da dependência química. Em $21,7 \%(n=76)$ dos casos, houve a presença de prejuízos laborais, desses, $63,2 \%(n=50)$ já perderam o emprego em decorrência da dependência química. Por fim, $10,6 \%(n=37)$ já moraram na rua por consequência da dependência química.

Na tabela 2 são apresentados os dados relacionados ao envolvimento dos dependentes químicos com a criminalidade e com a violência. Entre eles, $20,6 \%(n=72)$ cometeram algum tipo de infração penal, entre as quais destacam-se em $49 \%(n=35)$ furtos e $13 \%(n=9)$ tráfico de drogas. Em $29 \%$ ( $n=21)$ houve como consequência a privação de liberdade.
No que se refere à violência, $26,6 \%(\mathrm{n}=93)$ praticaram algum tipo sendo que desse, $63 \%(n=59)$ cometeram agressão verbal, e física $37 \%(n=34)$. Vale destacar que a violência física não foi considerada ato criminal pelos pacientes como prescrito no artigo 129 do Código Penal Brasileiro.

Tabela 1 - Prejuízos familiares, ocupacionais e sociais decorrentes do uso abusivo de drogas. Curitiba, 2011

\begin{tabular}{lcc}
\hline Abandono estudos & $(\mathbf{n = 3 5 0 )}$ & $\mathbf{( \% )}$ \\
Sim & 23 & $6,6 \%$ \\
Não & 02 & $0,6 \%$ \\
Problemas Familiares & & \\
Sim & 189 & $54 \%$ \\
Não & 17 & $4,9 \%$ \\
Problemas Conjugais & & \\
Sim & 103 & $29,4 \%$ \\
Não & 02 & $0,6 \%$ \\
Problemas Laborais & & \\
Sim & 76 & $21,7 \%$ \\
Não & 03 & $0,9 \%$ \\
Morar na Rua & & \\
Sim & 37 & $10,6 \%$ \\
Não & 09 & $2,6 \%$ \\
\hline
\end{tabular}

Tabela 2 - Envolvimento dos dependentes químicos com criminalidade e violência. Curitiba, 2011

\begin{tabular}{lcc}
\hline Criminalidade & $\mathbf{( n = 3 5 0 )}$ & $\mathbf{( \% )}$ \\
Sim & 72 & $20,6 \%$ \\
Não & 25 & $7,1 \%$ \\
Violência & & \\
Sim & 93 & $26,6 \%$ \\
Não & 08 & $2,3 \%$ \\
\hline
\end{tabular}

\section{DISCUSSÃO}

O consumo de múltiplas drogas é uma característica da atualidade, que paulatinamente substitui o uso exclusivo. Entre as diversas motivações para esse modo contemporâneo, está a necessidade de manipular a intensidade ou o efeito da droga de preferência, na intenção de minimizar as consequências da abstinência ou de potencializar e prolongar seus efeitos ${ }^{(7-8)}$.

À exemplo pode-se citar o consumo do álcool após o uso abusivo do crack, com a intenção de abrandar os efeitos negativos provocados pela síndrome de abstinência e, também a maconha consumida concomitante ao crack (mesclado) que prolonga a duração do efeito do $\operatorname{crack}^{(8)}$. No entanto, vale ressaltar que o 
uso de múltiplas drogas dificulta a adesão do indivíduo no tratamento, bem como prejudica o sucesso das intervenções terapêuticas, contribuindo para repetidos episódios de recaída ${ }^{(7-9)}$.

O elevado número de pessoas que foram internadas em decorrência do uso de álcool, destacado no presente estudo, coaduna que este se configura entre os mais comuns transtornos mentais consequente de substâncias psicotrópicas na atualidade, que independe da etnia, gênero ou de condições sociais. Isso ocorre porque o álcool está presente em meio a confraternizações e a rituais religiosos, caracterizando um importante simbolismo cultural em nossa sociedade ${ }^{(8-9)}$.

O alcoolismo é um quadro complexo de difícil definição entre o beber socialmente e o quadro patológico, estes limites podem ser estabelecidos pela frequência de consumo, quantidade de álcool ingerido e pela compulsão pela substância, levando em consideração problemas individuais, ocupacionais, familiares e sociais ${ }^{(1)}$.

A dependência ao álcool se instala de maneira insidiosa e lenta, prejudica a qualidade de vida, ocasiona perdas de oportunidades, impossibilidade de continuidade dos estudos e dificulta o acesso ao mercado de trabalho, situações que estão diretamente relacionadas com a criminalidade ${ }^{(9)}$.

A tendência mundial do consumo de drogas é cada vez mais precoce, geralmente na adolescência, principalmente do álcool, droga lícita e, consequentemente, a substância psicoativa mais consumida. Os púberes, com a personalidade em desenvolvimento, em geral, buscam novas sensações e experiências tornando-se mais suscetível a experimentação por influências externas $^{(10)}$. Comumente essas influências provêm da pressão exercida por um grupo de amigos e da mídia que transpassa o consumo de drogas como uma prática prazerosa $^{(10)}$. Uma pesquisa com dependentes de drogas em tratamento apresentou que a influência exercida pelos pares para o início do uso chega a $75 \%$ dos casos, desses, em média $19 \%$ o fizeram como motivo para aceitação do grupo de $\operatorname{amigos}^{(5)}$.

A baixa escolaridade dos participantes desta pesquisa pode estar relacionada ao início precoce do uso de drogas. Há que se considerar que os púberes não levam em consideração que os transtornos mentais, em decorrência do abuso de drogas, exercem considerável impacto negativo sobre sua vida, pois as consequências do transcendem a adolescência e atinge a fase adulta desfavorecendo uma geração economicamente ativa, o que sugere uma incapacidade produtiva importante provocada pelo abuso de drogas ${ }^{(11)}$.
Dentre os motivos que levam a desenvolverem problemas de ordem familiar, em consequência do uso compulsivo de drogas, estão as perdas do vínculo familiar e as dificuldades de relacionamento com pai, mãe, irmãos ou esposa ${ }^{(12)}$.

Considerando o aumento significativo do consumo de drogas nas últimas décadas, ressalta-se que a família sofre as sérias consequências diante desse quadro. Isso porque, na maioria das vezes, os familiares não entendem o processo de adoecimento do dependente, apresentam dificuldades no manejo das atitudes e buscam ingenuamente, de maneira ansiosa e precipitada, a resolução do problema ${ }^{(13)}$.

A dependência química produz um quadro de patologias que favorece situações desagradáveis, conflitos e crises nos relacionamentos interpessoais, causando dificuldades no manejo dessa problemática entre os familiares. No entanto, mesmo diante do quantitativo alarmante de dependentes, a família ainda é uma temática pouco estudada por especialistas ${ }^{(13)}$.

Em decorrência do comportamento compulsivo, em geral as cônjuges de dependentes químicos tomam uma postura com excessivas cobranças e desconfianças que também contribui para o comportamento agressivo do dependente químico e aumento do consumo de drogas, favorecendo repetidas discussões e, consequentemente, separações conjugais ${ }^{(12)}$.

O consumo abusivo de drogas pode gerar problemas laborais e, muitas vezes, levar ao desemprego. Sabe-se que o baixo nível de lucidez pelo efeito da droga no sistema nervoso central produz um quadro de desorganização e compromete o desenvolvimento de atividades pertinentes a função no trabalho, como também gera absenteísmos e, por consequência, a perda do trabalho ${ }^{(6)}$.

Este fato corrobora com estudo realizado com dependentes químicos em tratamento em uma unidade de reabilitação, o qual apontou que $77 \%$ dos indivíduos perderam o emprego ao menos uma vez devido ao envolvimento com drogas ${ }^{(14)}$. Outros estudos apontaram esse mesmo perfil em mais de $50 \%$ dos dependentes de drogas ${ }^{(6,11)}$.

É comum o usuário tentar manter-se no emprego mesmo diante dos efeitos nocivos da dependência, omitindo sua condição e seu comportamento vicioso com receio de represália e perda do emprego. Entretanto, o uso de drogas passa a ser prioridade para o dependente e, em decorrência do tempo gasto para a obtenção e consumo da droga, do tempo que o efeito da droga permanece no organismo, tais condições interferem 
de maneira significativa no desempenho profissional. É importante ressaltar que esta prática posterga um eventual tratamento ${ }^{(6)}$.

Morar na rua em algum momento da vida foi um dos dados relevantes no presente estudo que corrobora com uma pesquisa realizada com usuários de substâncias psicoativas internados para o tratamento da dependência em um hospital psiquiátrico de Porto Alegre-Rio Grande do Sul. Este apontou que mesmo tendo moradia própria, cerca de $30 \%$ dos casos já moraram na rua em algum momento de suas vidas em decorrência da dependência ${ }^{(15)}$.

Esta situação ocorre porque, geralmente, o uso de drogas está associado a diversos conflitos entre o usuário e os familiares, e a decisão pela continuidade do envolvimento com drogas implica em sair de casa e morar na rua ${ }^{(16)}$. Outra condição comum que leva o dependente químico a sair de casa e permanecer em situação de rua são as constantes ameaças de morte feitas por traficantes em decorrência da falta de pagamento da droga ${ }^{(15,17)}$.

O envolvimento com a criminalidade também é um fator intrinsecamente relacionado com a dependência química, há maior risco de cometer delitos entre a população que possui algum dependente de substância psicoativa que naquelas que não possuem ${ }^{(18)}$.

A partir da década de 1980 houve aumento considerável da criminalidade relacionado ao uso de drogas, entre eles o roubo, sequestro e homicídio. Nesse mesmo período, o tráfico de drogas foi responsável por três vezes mais condenações criminais que há 20 anos $^{(12)}$. Atualmente, sabe-se que todo dependente químico é potencialmente um pequeno traficante ${ }^{(5)}$, pois a venda de drogas oferece, por meio do trabalho não formal, um ideal de conquista que visa a remuneração fácil e o prestígio, como meio de afastar-se de uma realidade de vida precária que muitos levam ${ }^{(18)}$.

Nesse caso, a vontade de consumir drogas é sempre maior que a prudência. A forte relação entre consumo de drogas e criminalidade se tornou evidente na realidade brasileira, e esta condição tem trazido diversos problemas sociais exigindo remodelação conceitual e estrutural do sistema judiciário criminal e dos serviços de saúde, incluindo a interação entre esses dois serviços ${ }^{(18)}$.

Há correlação positiva e significativa entre a idade do primeiro uso com a do primeiro crime, que indica que ao momento que de início com as drogas, lícitas ou ilícitas, o mesmo ocorreu também na inserção ao mundo do crime. Isto sugere que a ausência na escola está diretamente envolvida com comportamentos de risco, entre eles, a delinquência ${ }^{(18)}$.
Esse fato corrobora com estudo realizado com 30 indivíduos em tratamento para a dependência química, o qual destacou que $60 \%$ dos participantes já cometeram algum crime em decorrência do uso de drogas, seja para a aquisição ou sob o efeito das mesmas. Desses, $40 \%$ possuíam antecedentes criminais e 33\% já estiveram presos. A média de escolaridade nesse mesmo estudo não passou de 9,4 anos e todos os crimes foram cometidos após o início do consumo de drogas ${ }^{(15)}$. É importante ressaltar que encontramos esse mesmo aspecto na presente pesquisa.

Os altos níveis de violência como um todo têm sido considerado pela Organização Mundial da Saúde um problema de saúde pública; o aumento da violência, principalmente a doméstica tem ocorrido em maior incidência naquelas famílias que há um dependente químico ${ }^{(18)}$.

Uma pesquisa realizada com o intuito de identificar os tipos de violência ocorrida em domicílios em que reside ao menos um dependente químico identificou que a maioria era agressões verbais e, entre as mais comuns destacaram a discussão $(81,8 \%)$, escândalos $(70,9 \%)$ e ameaças de quebrar objetos (38,7\%). As agressões físicas apareceram em 46,2\% dos casos. Ainda de acordo com o referido estudo, em mais da metade dos casos o agressor estava sob o efeito da droga e, quanto maior foi o período de consumo, maior o impacto da violência, na qual a parceira conjugal acaba sendo o principal alvo das agressões ${ }^{(19)}$.

A proporção de atos violentos aumenta à medida que o indivíduo utiliza o álcool ou outras drogas. Fatores orgânicos, socioculturais e de personalidade estão envolvidos na prática da violência; a probabilidade de cometer algum ato agressivo aumenta à medida que a síndrome de abstinência surge. Há alta carga emocional e psicoafetiva envolvendo os dependentes, gerando diversos conflitos permeados por insultos, desabafos, agressividade verbal e física, seja na residência, ou na via pública ${ }^{(18)}$.

O dependente químico, em geral, apresenta sentimentos de menos valia, dificuldades em superar os obstáculos e problemas de interação e comunicação, com isso se angustiam expressando suas emoções de maneira impulsiva e descontrolada ${ }^{(4,18)}$. Destarte, apresentam conduta agressiva, com pouca sensibilidade e afeição no relacionamento com outro indivíduo, e certa irritabilidade. Assim, da mesma forma que as atitudes criminais devem ser levadas em consideração, os efeitos de cada substância, aspectos sociais, culturais e de personalidade também devem ser considerados, haja vista que interferem diretamente em uma conduta violenta ${ }^{(18)}$. 
Um pesquisa realizada com o intuito de comparar traços de personalidade de dependentes e não dependentes químicos destacou que $69 \%$ daqueles que tinham envolvimento com drogas apresentavam labilidade, insegurança e dificuldade de adequar-se ao meio em que vivia. Ainda relacionado à pesquisa, $38,4 \%$ dos participantes externavam sentimentos de menos valia e dificuldade para superar desafios, $30,7 \%$ dificuldade de comunicação e $23 \%$ dificuldade de tomada de decisão. Características que podem induzir a impulsividade e agressividade e diferem daqueles que não estavam envolvidos com as drogas ${ }^{(4)}$.

\section{CONCLUSÕES}

Conclui-se com esta pesquisa que o impacto social provocado pelo uso de drogas atinge todas as esferas da vida do indivíduo. No núcleo pessoal, a maioria dos dependentes iniciaram o uso de drogas na adolescência e como consequência, abandonaram os estudos, tiveram problemas familiares e no trabalho.

Os dados desta pesquisa demonstram que é necessário adequar e investir em propostas para a reinserção social e profissional de dependentes químicos. Este transtorno age rapidamente na exclusão do funcionamento social do indivíduo, pois diversas habilidades que foram perdidas, ou não adquiridas ao longo do tempo, devem ser reabilitadas.

Deve-se investir em ações preventivas que abordem aqueles com idades anteriores à média de idade citada no estudo, para que a vida produtiva desses jovens não se torne prejudicada por consequência da dependência química. Compreende-se que não se trata de uma proposta fácil de ser realizada, mas fundamental para o sucesso do tratamento.

\section{REFERÊNCIAS}

1. Delucia R, Oliveira Filho RM. Farmacologia Integrada. $3^{\mathrm{a}}$ ed. Rio de Janeiro: Revinter; 2007.

2. Oga S, Camargo MMA, Batistuzzo JAO. Fundamentos de toxicologia. $3^{a}$ ed. São Paulo: Ateneu; 2008.

3. UNODC - United Nations Office on Drugs and Crime. World drug report. New York: United Nations Office on Drugs and Crime; 2012.

4. Rocha JCG, Rocha Junior A. Aspectos de personalidade observados emumaamostra deindivíduosusuários de drogas por meio do teste Wartegg. Revista Saúde. 2010;4(2):10-22.
5. Tuller NGP, Rosa DTM, Polli MCS, Catelan-Mainardes SC. Os sofrimentos e danos biopsicossociais de dependentes químicos em recuperação. Revista Cesumar. 2009;14(1):137-74.

6. Beck LM, David MSL. O abuso de drogas e o mundo do trabalho: possibilidades de atuação para o enfermeiro. Esc. Anna Nery. 2007;11(4):706-11.

7. Oliveira LG, Nappo SA. Caracterização da cultura de crack na cidade de São Paulo: padrão de uso. Rev. Saúde Públ. 2008;42(4):664-71.

8. Ferreira ACZ, Capistrano FC, Maftum MA, Kalinke LP, Kirchhof ALC. Caracterização de internações de dependentes químicos em uma unidade de reabilitação. Cogitare enferm. 2012;17(3):444-51.

9. Miranda FAN, Simpson CA, Azevedo DM, Costa SS. O impacto negativo dos transtornos do uso e abuso do álcool na convivência familiar. Rev. Eletr. Enf. [Internet] 2006;8(2) [acesso em 23 mai 2011]. Disponível: http:// www.fen.ufg.br/revista/revista8_2/v8n2a07.htm

10. Pechansky F, Szobot CM, Scivoletto S. Uso de álcool entre adolescentes: conceitos, características epidemiológicas e fatores etiopatogênicos. Rev. Bras. Psiquiatr. 2004;26(1): 14-7.

11. Peixoto C, Prado CHO, Rodrigues CP, Cheda JND, Mota LBT, Veras AB. Impacto do perfil clínico e sociodemográfico na adesão ao tratamento de pacientes de um Centro de Atenção Psicossocial a Usuários de Álcool e Drogas (CAPSad). J. Bras. Psiquiatr. 2010;59(4):317:21.

12. Souza J, Carvalho AMP. Filhos adultos de pais alcoolistas e seu relacionamento na família de origem. Saúde de Transformação Social. 2012;3(2):43-51.

13. Vargens OMC, Brands B, AdlafE, Giesbrecht N, Simich L, Wrigth MGM. Uso de drogas ilícitas e perspectivas críticas de famílias e pessoas próximas, na cidade do Rio de Janeiro, zona norte, Brasil. Rev. Latino-Am. Enfermagem. 2009;17(Esp.): 776-82.

14. Silva LHP, Borba LO, Paes MR, Guimarães NA, Mantovani MF, Maftum MA. Perfil dos dependentes químicos atendidos em uma unidade de reabilitação de um hospital psiquiátrico. Esc. Anna Nery. 2010;14(3):585-90.

15. Guimarães CF, Santos DVV, Freitas RBA. Perfil do usuário de crack e fatores relacionados à criminalidade em unidade de internação para desintoxicação no 
Hospital Psiquiátrico São Pedro em Porto Alegre (RS). Rev. psiquiatr. Rio Gd. Sul. 2008;30(2):101-8.

16. Souza FM, Valencia E, Dahl C, Cavalcanti MT. A violência urbana e suas consequências em um centro de atenção psicossocial na Zona Norte do Município do Rio de Janeiro. Saude soc. 2011;20(2):363-76.

17. Martins $\mathrm{MC}$, Pillon SC. A relação entre a iniciação do uso de drogas e o primeiro ato infracional entre os estudantes em conflito com a lei. Cad. Saúde Pública. 2008;24(5):1112-20.

18. Chalub M, Telles LEB. Álcool, drogas e crime. Rev. Bras. Psiquiatr. 2006;28(2):69-73.

19. Fonseca AM, Galduróz JCF, Tandowski CS, Noto AR. Padrões de violência domiciliar associada ao uso de álcool no Brasil. Rev. Saúde Públ. 2009;43(5):743-9. 